\title{
The Research Status of the Wetting of Metal/Ceramic in the High Temperature Self-Lubricating Materials
}

\author{
Li Chao", Wang Yan Jun ${ }^{1, ~ *, ~ Q i n ~ K e ², ~ L i ~ S h u ~ L o n g ~}{ }^{1}$ \\ ${ }^{1}$ School of Mechanical Engineering, University of Jinan, Jinan, China \\ ${ }^{2}$ Shandong Machinery Industry Association, Jinan, China \\ Email address: \\ 1600359600@qq.com (Li Chao),me_wangyj@ujn.edu.cn (Wang Yan Jun), qinke227@sina.com (Qin Ke), \\ 1101701864@qq.com (Li Shu Long) \\ ${ }^{*}$ Corresponding author
}

\section{To cite this article:}

Li Chao, Wang Yan Jun, Qin Ke, Li Shu Long. The Research Status of the Wetting of Metal/Ceramic in the High Temperature Self-Lubricating Materials. International Journal of Materials Science and Applications. Vol. 5, No. 2, 2016, pp. 108-112. doi: 10.11648/j.ijmsa.20160502.22

Received: April 11, 2016; Accepted: May 5, 2016; Published: May 9, 2016

\begin{abstract}
The wettability of solid lubricant and matrix sintered body is very important for the production of the high temperature self-lubricating composite material. The wettability of the metal/ceramic is ubiquitous phenomenon in materials science and people have long the research in this field. Research on the wettability of the ceramic/metal has important practical significance for developing new type of metal/ceramic systems, exporing and developing the preparation technology of material, and preparing high performance of metal/ceramic composite materials. In this paper, from the wetting phenomenon of the metal/ceramic, the commonly used theory of the wettability research is stated, various experimental methods of wettability are introduced, and the significant importance of improving the wettability for developing metal ceramic friction material is indicated. What's more, some methods of improving the wettability are proposed.
\end{abstract}

Keywords: High Temperature Self-Lubrication, Wettability, Metal/Ceramic

\section{Introduction}

From the physics point of view, the study of material list, interfacial phenomena has great significance. Unless it is in the vacuum state, because the material is always contacting with different media (solid, liquid, gas) through the surface, thus the performance of various materials and manufacturing processes (such as corrosion, aging, hardening, destruction, printing, coating, adhesive, composite, etc.) are strongly influenced by surface characteristics. The wetting of liquid to solid is a common interface phenomenon. The wetting phenomenon not only affects the various life activities of animals and plants in the nature, but also plays an important role in the human life and production. For instance, printing of the plastic surface, cementation of the polymers and other materials, lubrication of the machinery, as well as decontamination, emulsification, dispersion, etc., are all closely related to wetting phenomenon.

As we all know, when preparing the metal matrix high temperature self-lubricating composite materials by using the liquid phase process, the wettability between reinforcement and matrix is an important factor that must be considered [1-2]. The wettability of metal alloy and ceramic will directly affects the material's caking property. If the wettability is better, the material will more compact and the property will more excellent [3]. And the wettability is good or bad directly affect whether reinforcement could evenly distribute in liquid metal, the size of the pressure in the process of squeeze casting and the infiltration of the liquid metal substrate to ceramic reinforced precast blocks, which largely determines to the difficulty of the composite material preparation and even success [4]. Besides, the wettability and interface chemical features of metal matrix and reinforcement determine the quality of the interface bonding in the very great degree, and then affect the final performance of composite materials. From this, we can see that in the preparation and development process of high temperature metal matrix self-lubricating composite material, systematically study and analysis is very important to the wettability and interface chemical characteristics of alloy melt and ceramic reinforcement. 
The metal matrix composites has been widely researched and developed in the past forty years. The research of the wetting behavior of strengthen body and matrix could provide effective guidance for the preparation of composite materials [5]. If the wettability between the strengthen body and matrix is very poor, it will hard to make it effective compound without improve the wettability between them. The wettability on the surface of the material has been a large number of used in materials engineering. For example, the high-temperature self-lubricating composite material generates lubrication film in the friction surface by taking advantage of the wettability of lubricant to surface of substrate to realize the compensation lubrication [6]. As a result, this article through introduce and state the related theory and methods of wettability, it hopes to be able to provide theoretical basis for improving the wettability of metal and ceramics. What's more, by controling the wettability of the material surface to provide reference for the realization of high temperature self-lubrication in special conditions and so as to preferably play to the use of material value.

\section{Wetting Phenomenon}

Wetting effect actually involved in gas, liquid and solid phase. Because of solid surface's irregularity and solid surface energy could not be directly measured, and molecular structure of liquid is less tidy than solid, and it's molecular spacing is small than gas, the molecular inter-atomic forces must consider, this makes the solid-liquid-gas three-phase interface is very complex.

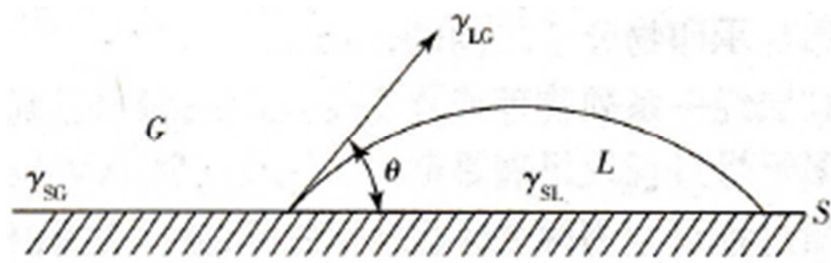

Figure 1. Contact Angle.

Make a droplet drops on the solid surface, forming the shape of figure 1 . In the solid-liquid-gas three phase interface, the interfacial tension in solid-gas is $\sigma_{S G}$, the interfacial tension in solid-liquid is $\sigma_{S L}$, the interfacial tension in gas-liquid is $\sigma_{L G}$. The angle in three-phase junction from the solid-liquid interface passes through the liquid internal to the gas-liquid interface is called contact angle, angle to $\theta$. So three-phase interfacial tension namely $\sigma_{S G}, \sigma_{S L}, \sigma_{L G}$ generally obey the Young's equation:

$$
\sigma_{S G}=\sigma_{S L}+\sigma_{L G} \cos \theta
$$

The equation is the basis of the study of liquid-solid wetting effect [7]. In general, $\theta^{\prime} s$ size is a criterion that determine the size of the wettability is good or bad. If:

$$
\theta=0 ; \cos \theta=1
$$

Fully wetting, liquid spread out on solid surface;

$$
0<\theta<90^{\circ}
$$

Liquid could wetting solid, and $\theta$ is smaller, the wettability is better;

$$
90<\theta<180^{\circ}
$$

Liquid could not wet solid;

$$
\theta=180^{\circ}
$$

Fully not wetting, liquid condenses ball on the solid surface.

\section{The Classification of the Wettability}

Wetting phenomenon. According to the interface bonding, high temperature wetting system generally fall into two categories, the not-reaction system and reaction system.

The not-reaction system refers to the system that it is almost no change that solid surface properties before and after contact with the liquid. The wetting driving force in this kind of system mainly comes from thesystem interface's change that the liquid-solid and liquid-gas interface instead of solid-gas interface [8].

Tanner and De Gennes points out that the $n=3$ based on a analysis model that proposed by droplet spreading mainly controlled by its own viscous flow. Due to the fast viscous flow of liquid metal, so the metal droplet spreading time (millimeter size droplets to balance the time) is generally short, around $10^{-4} \sim 10^{-1}$ seconds (specific time mainly with the physical properties such as viscosity and surface tension of the metal as well as related to the size of the final equilibrium contact angle).

In the reaction system, the wetting process is usually accompanied by the occurrence of the phenomenon such as substrate solubility, activity element diffusion and adsorption, and chemical reaction and it's formation of reaction products. So droplets spread out mainly controlled by the reaction factors rather than by the viscous flow control. Compared with the not-reaction system, reaction system's balance time is longer, in about $10^{-1} \sim 10^{-4}$ seconds.

\section{Common Theories in Wettability Research}

There are three classical theories in wettability research: theory of Young (Fig. 2) [9], Wenzel theory (Fig. 3) and Casssie Baxter theory (Fig. 4).

$$
\begin{gathered}
\cos \theta=\left(\gamma_{s c}-\gamma_{s L}\right) / \gamma_{l G} \\
\cos \theta_{w}=r\left(\gamma_{s c}-\gamma_{s L}\right) / \gamma_{l G}=r \cos \theta \\
\cos \theta_{c}=f_{1}\left(\gamma_{s G}-\gamma_{s L}\right) / \gamma_{L G}=f_{1} \cos \theta-f_{2}
\end{gathered}
$$

Young theory thinks that the contact angle that liquid on the solid surface just related to the surface tension of solid-gas, 
solid-liquid and liquid-gas interface for ideal smooth surface of the solid, and content Eq. 6. The formula shows that despite the reduction of solid surface can improve the lyophobic sex, increase the contact angle, but only rely on the lower surface energy method can't make smooth surface to obtain super lyophobic properties [10]. And it also shows that smooth surface could obtain the ultra lyophilic sex, but only for parts material and the liquid. Most of the material (especially metal)'s smooth surface does not display the super lyophilic sex.

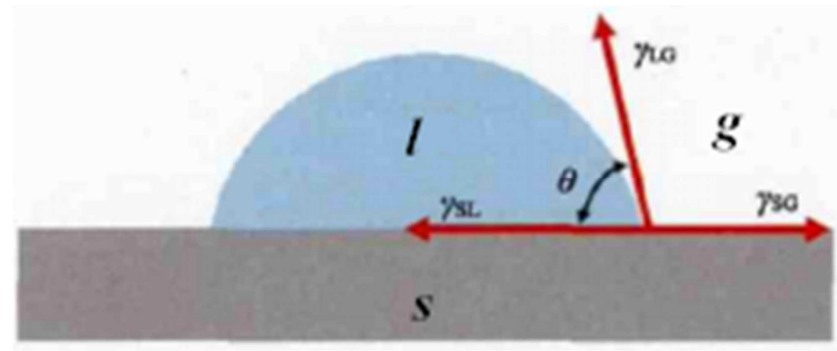

Figure 2. Schematic of a water droplet on a solid surface.

Wenzel theory thinks that the actual surface smooth surface is not ideal, there is a micro rough geometry structure, the liquid's contact angle that on it not only related to the surface tension of solid-gas, solid-liquid and liquid-gas interface, but also relatedto microstructure, and satisfy the Eq. 7. The formula shows that the micro rough structure can make the lyophobic surface become more lyophobic, thus obtain the ultra thin liquid, also can make the liquid surface become more kiss and then obtain the ultra lyophilic.

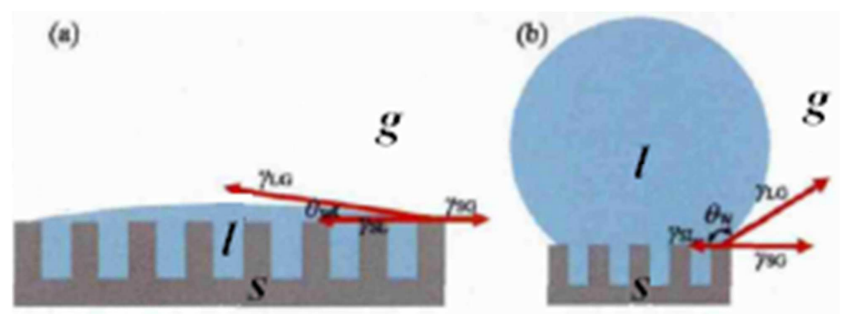

Figure 3. Schematic of a water droplet with Wenzel state on a rough solid surface.

Although Wenzel theory can explain why the contact angle of liquid on a solid surface minimax, but fails to explain why some surface rendering with minimal rolling angle of the super lyophobic sex, the liquid easily finishes in some surface. Therefore, Cassie and Baxter proposed another thory (i.e., Cassie-Baxter theory) to explain the phenomenon that Wenzel theory could not explain. The theory thinks that the contact between liquid and ough solid surfaces that has micro structure is compound contact, there is gas and solid at the bottom of the liquid and the contact angle of liquid on it not only related to he surface tension of solid-gas, solid-liquid and liquid-gas interface, but also relate to the proportion that solid-liquid interface and gas-liquid interface in the composite interface and satisfy the Eq. 8 [11]. The formula shows that The formula shows that by reducing the contact area of solid-liquid can increase the contact angle, reduce the adhesion force between the liquid and solid, and could contribute to the formation of small rolling angle.

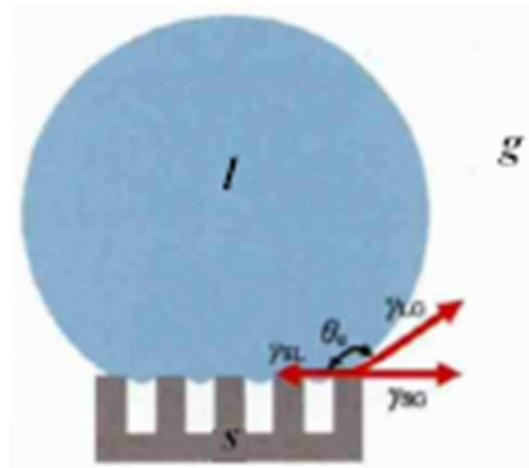

Figure 4. Schematic of a water droplet with Cassie-Bwxter state on a rough solid surface.

\section{Wettability Experiment Methods}

\subsection{A Drop Method}

The traditional method of measuring the wettability of metal/ceramic is a drop method, this method also is the most widely used experimental method. Its principle is that melting the metal on ceramic substrate surface, by observing the camera, measuring the contact angle on the photos or projection screen, or measuring the contact angle and the cooled metal droplet shape after metal condensation based on the Young's equation. A drop method commonly used in 400 2000. The method is convenient and quick, data precision, general error in the plus or minus $\pm 1^{\circ}$. More important is the method could dynamically observe the process that metal droplet e melt and spread out on the surface of the ceramic. The contact angle measurement methods are: (1) direct measurement method; (2) measuring size of droplet shape, through the Laplace equation to calculate the contact angle $\theta$, the method's calculation is trival, only suitable for $\theta$ is larger; (3) curve fitting, the method by measuring near angle curve coordinate values, with curve fitting extrapolation to three-phase intersection, then calculate $\theta$, so gains the calculated value of precision is higher. Because of a drop methodis strongly influenced by experimental condition in the measurement process, so strictly control condition parameters is the key to obtain accurate results [12].

\subsection{Micro Drop Method}

Because of the wettability's measurement accuracy of easy oxidation of metal and the system that exists in nterface reaction is not high by a drop method, so develop the micro drop method. By evaporation and sputtering layer of metal to the surface of the ceramic substrate, heating the metal under vacuum, forming droplet on ceramic surface after melting, then measuring the contact angle. Due to this method avoids the effects of Alumina membrane layer on contact angle, so the angle's measurement is more accurate. Literature [13] pointed out that: this method is difficult to measure the dynamic contact angle, and the operation is more difficult. 


\subsection{Immersion Method}

The disc or cylindrical that made by immersing ceramic in the metal liquid, measuring the quality of the ceramic and recording the curved surface shape closed to the edge of the ceramic, measuring $\sigma_{l s}$ and $\theta$, so measuring the wettability. But it is quite harsh for experimental conditions. I. Rivollee [14] et al. improved this kind of method that make the measurement of wetting angle at the same time also can measure the adhesion work. This method proved to be more precise, but the measuring device is more complex, and experimental conditions are relatively strict.

Moreover, in order to adapt to a variety of different wettability system, researchers have proposed many other experimental methods, such as capillary pressure method. The capillary pressure method is widely used for measuring the wettability of fibrous, granular ceramics and metal, it is to solve the wetting angle or adhesion by measuring the critical impregnation pressure. As the study of the extension and deepen constantly of wettability system, the new test method will be constantly emerging.

\section{The Main Technology of Improving the Wettability}

Further study of wetting has important guiding significance for the preparation of composite materials, and providing the feasibility plan for the wettability of metal to ceramic. To improve the wettability mainly from the following three aspects [15]: (1) Elevating the solid phase surface energy; (2) Reducing the solid/liquid interface energy; (3) Reducing the liquid surface tension. Now there are a lot of method that can effectively improve the wettability of metal, improve the interface bonding strength.

\subsection{Ceramic Surface Pretreatment}

Ceramic surface pretreatment by Young's equation: increasing $\sigma_{s g}$ can reduce the contact angle, and carrying on the surface treatment of ceramic particle. Through physical and chemical methods to increase $\sigma s g$, including physical vapor deposition (PVD), chemical vapor deposition (CVD), physical and chemical vapor deposition (PCVD), physical and chemical cleaning (with some salt, such as sodium carbonate, zirconium fluoride acid potassium, etc, to deal with $\mathrm{SiC}$ particles and remove oxide films and pollutants on the surface of the particles, can improve the wettability of $\mathrm{SiC}$ with liquid metal). To preheat the ceramic particles can reduce or eliminate the impurities and gas of surface adsorption, and improve its wettability with liquid metal.

\subsection{Increase Wetting Coating}

Reinforcing coating treatment is a kind of effective way that improving the wettability. Ceramic surface metal coating can improve the solid surface energy, replace associativity poor interface with a new form of metal/ceramic interface could improve the wettability. $\mathrm{Ni}$ and $\mathrm{Cu}$ is the most commonly used metal coating material. We can take Chemical vapor deposition method and spray $\mathrm{Ni}, \mathrm{NiN}$ or TiNi in the surface of $\mathrm{Al}_{2} \mathrm{O}_{3}$ ceramics to change wettability and enhance the interfacial bonding strength.

Using the coating technology effect is good, but the process is complex, high cost. What's more, reinforcing coating research is still in the experimental stage, the deep theoretical research is not enough. Therefore, it is need to further explore for the application and development of reinforcingcoating.

\subsection{Alloying}

Alloying approach is the most widely used means of improving wettability, and the effect mechanism of wettability of alloy element to elemen has been carried on the thorough research, the main mechanism is: (1) Alloying elements's adsorption and enrichment in liquid metal surface and solid/liquid interface could reduce the surface tension of liquid metal and solid/liquid interfacial tension; (2) Alloying elements's interface reaction on the surface of solid/liquid could form interface reaction products [16].

\subsection{Raise the Temperature}

In general, we can raise the temperature to reduce the surface energy of the metal liquid, so as to improve the wettability. When we prepare metal matrix wear resistant composite materials by casting and overheat the liquid metal could improve the wettability of the ceramic wear particles and metal substrate.

However, raise the temperature also has some limitations: (1) For non-reactive metal/ceramic system, the effect is not obvious. Literature [17] showed that for the aluminum/alumina system, temperature raises $1000^{\circ} \mathrm{C}$, contact angle only decreases $5 \sim 10^{\circ} \mathrm{C}$. (2) At high temperatures, which may be generate brittle phase at the interface, thereby reducing the performance of the composite materials. (3) When raising temperature, we must improve the high temperature resistant performance of containers, tools, etc, but increase the cost. Therefore, we should give full consideration to all aspects of the situation and consider advantages and disadvantages and choose the appropriate composite temperature.

\subsection{Ultrasonic Vibration}

Due to the adsorption gas, impurities in reinforced ceramics surface, so forming a dirt layer of surface and causing the decrease of surface properties of the ceramic and the wetting ability of ceramic/metal. If we take appropriate ultrasonic frequency, there will produce cavitation phenomenon. Cavitation could damage and remove the surface of the fiber fouling layer and the oxide film and decrease wetting angle of system and improve the wetting ability between ceramic and metal. Ultrasonic temperature effect also reduces molten surface properties and viscosity, thus improving the wettability of molten to ceramics.

Reinforce metal melt's dynamics. We could make the ceramic particles that it's wettability with metal is not strong 
uniformly distributed in the matrix by fluid dynamics effect after metal melt mixing. In the semi-solid casting process, strong mechanical stirring makes the liquid metal through the enhanced particle surface at high shear rate, it can effectively improve the wettability of metal/ceramic.

In addition, using the appropriate protective atmosphere and fluid dynamics method could obtain good wettability.

\section{Conclusion}

The bonding strength of metal and ceramic has a significant impact on the using performance of material in the high temperature self-lubricating composite materials, and the wettability of metal and ceramic has close relation with its bonding strength, and it has vital significance in preparation of low friction metal matrix composites that contains other ceramic phases and friction function coating. Improving the wettability of metal and ceramic and making metal ceramic material that has good comprehensive performance, will be conducive to obtain better performance, longer life casting materials, high-temperature self-lubricating sinter material and cutting tool, and will promote the industrial application of metal ceramic friction materials.

Metal/ceramic composite material's performance are superior, it has high strength, high specific modulus, low thermal expansion, heat-resisting, wear-resisting, conductive, thermal conductivity and other excellent performance and has a broad application prospect. It is a kind of high-tech materials that is developing and also is a focus that studied in the future. At present, the research of metal/ceramic composite materials is still in its infancy, there is no mature theory system about wettability and we need further exploration to seek appropriate experimental method in order to furture study the mechanism of metal ceramic wetting. The Wettability of metal and ceramic reinforced phase is one of the important factors that determines the success or failure of composite materials technology and material performance. Once wettability problems are resolved, metal/ceramic composite materials that have high quality and low price will be gained extensive development and application.

\section{Acknowledgements}

The authors would like to thank the National Natural Science Foundation of P.R.China for the financial support (ID: 41108336).

\section{References}

[1] DELANNAY F, FRO YEN L, DERUYTTERE A. The wetting of solids by moltenmetals and its relation to the preparation of metal-matrix composites composites [J]. Journal of Materials Science. 1987, 22(1): 1-16.
[2] ASTHANA R. Reinforced cast metals: Part II Evolution of the interface [J]. Journal of Materials Science. 1998, 33(8): 1959-1980.

[3] X. Z. Yan, W. S. Liu, N. X. Fang, etc. Development in metal encapsulating ceramic matrix composites $[\mathrm{J}]$. Light Industry Machinery. 2015, 33(6): 1-4.

[4] YE H Z, LIU X Y. Review of recent studies in magnesium matrix composites [J]. Journal of Materials Science. 2004, 39(20): 6153-6171.

[5] X. B. Liu. Effect of electric current on the wetting and interfacial reaction between liquid and solid metel [D]. Shen Yang Institute of Aeronautical engineering. 2010, 18-22.

[6] Y. J, Wang. Study on fabrication, charaterization and tribological properties of high temperature self-lubrication metal ceramics with sweat gland structure [D]. Wuhan university of technology. 2008, 10-11.

[7] Z. F. Hu, G. R. Chen, Y. J. Du. Material Surface Interface. Shanghai: East China university of science and technology press [M]. 2007, 51-60.

[8] Y. Liu, J. B. Zhang, Y. Li, etc. The status and progress of MAX/matrix self-lubricating composite [J]. Materials Review: nanotechnology and new materials, 2015, 29(2): 517-523.

[9] Young T. An essay on the cohesion of fluids [J]. Philosophical Transactions of the Royal Society of London. 1805, 95: 65-87.

[10] Nishino T, Meguro M, Nakamae K, et al. The lowest surface free energy based on $\mathrm{CF}_{3}$ alignment [J]. Langmuir. 1999, 15(13): 4321-4323.

[11] J. L. Song, Y. Lu, Sh. Huang, X. Liu, W. J. Xu. Progress on research and application of extreme wettability surfaces [J]. Science and Technology Herald. 2015, 33(15).

[12] M. H. Chen, N. Liu, Y. D. Xu. The research status of metal/ceramic wettability [J]. Cemented Carbide. 2015, 19(4): 199-205.

[13] K. H. Chen, C. X. Bao, H. W. Liu. The wettability of metal/ceramic [J]. Materials Science and Engineering. 1997, 15(3): 6-10.

[14] Rivollee I. Charain D. Sinmi taneous measurement of contact angles and work of adhesionin metal-ceramic systems by immersion-emerson technique [J]. J. Mater Sci. 1990, 16(25): 3179-3185.

[15] I. A. Ibrahim, F. A. M ohamed, E. J. Lavernia, Particulate reinforced metal matrix composites-a review [J], J. M ater. Sci., 1991, 26: 1137-1156.

[16] J. Li, B. K. Gong, Q. S. Sun. The wettability of metal/ceramic. Shandong Metallurgy [J]. 2007, 29(6).

[17] X. F. Zhang, D. J. Wang, S. R. Chen. The wettability change mechanism of the liquid aluminum and ceramic. Powder Metallurgy Technology [J]. 2003, 21(1): 42-45. 